\title{
Coexistence of gastric gastrointestinal stromal tumor, intro-abdominal and retroperitoneal liposarcomas -a case report
}

\author{
Yong Zhou ${ }^{1}, \mathrm{Xu}$-Dong $\mathrm{Wu}^{2^{*}}$, Quan Shi ${ }^{3}$, Chuan-hai $\mathrm{Xu}^{4}$ and Jing Jia ${ }^{5}$
}

\begin{abstract}
Background: Gastric gastrointestinal stromal tumor (GIST), intro-abdominal and retroperitoneal neoplasms are distinct tumors arising from different cell layers; therefore, coexistence of such tumors is relatively rare.

Case presentation: A man complained of early satiety for 2 mouths, whose upper gastrointestinal (GI) endoscopy showed a tumor arising from the greater curvature of gastric body and extending into the lumen. Abdominal computed tomography (CT) revealed coexistence of gastric, intro-abdominal and retroperitoneal masses. Wedge resection for gastric tumor, resection for intro-abdominal and retroperitoneal tumors were done. The postoperative histological examination suggested simultaneous development of a gastric GIST, intro-abdominal and retroperitoneal myxoid liposarcomas.
\end{abstract}

Conclusion: Although both GISTs and liposarcomas originate from mesenchymal tissues, simultaneous development of a gastric GIST, intro-abdominal and retroperitoneal liposarcomas is the first such case to be reported in the literature.

Keywords: GIST, Intro-abdominal liposarcoma, Retroperitoneal liposarcoma, Myxoid liposarcoma

\section{Background}

Past decades have witnessed frequent cases of synchronous occurrence of a GIST and another neoplasms [1, 2]. However, gastric GISTs simultaneous development of a liposarcoma is rarely reported. Liposarcoma represents $20-30 \%$ of adult soft tissue tumors, most commonly -arising from the extremities, followed by the retroperitoneum [3]. Furthermore, its abdominal localization occurs only in $5 \%$ of cases [3]. Although both GISTs and liposarcomas originate from mesenchymal tissues, simultaneous development of a gastric GIST, intro-abdominal and retroperitoneal liposarcomas is extremely rare in the literature.

\section{Case presentation}

A 56-year-old male with early satiety for 2 mouths was admitted to our hospital. There was no history of weight loss, without relevant past and family history. An $18 * 25 \mathrm{~cm}$ oval tumor with medium texture was palpable below the left costal margin during the physical examination.

\footnotetext{
* Correspondence: hnjsycwxd@163.com

2Department of Gastroenterology, Yancheng City No.1 people's hospital, 16

Yuehe Road, Yancheng, Jiangsu Province 224005, China

Full list of author information is available at the end of the article
}

The routine biochemical and hematogical parameters were within normal limits, and tumour markers including CA-125, carcinoembryonic antigen (CEA) and CA19-9 levels were nothing special. Upper gastrointestinal endoscopy (GI) revealed a tumor arising from the greater curvature of gastric body and extending into the lumen (Fig. 1). Contrast enhanced CT scans of the abdomen showed a marked enhancement of polypoid mass protruding into the gastric lumen, a large poorly enhancing oval mass in the left abdomen and a heterogeneous round-like tumor adjacent to the left psoas (Fig. 1). Axial plain CT revealed the intro-abdominal tumor with CT values ranging from $-78 \mathrm{HU}$ (consistent with fatty tissue) to $27 \mathrm{HU}$ (related to the pancreas), with a mean CT value as $2 \mathrm{HU}$. However, the retroperitoneal tumor had $\mathrm{CT}$ values ranging from $-57 \mathrm{HU}$ (consistent with fatty tissue) to $18 \mathrm{HU}$ (related to the blood), with a mean CT value as $10 \mathrm{HU}$. The patient was diagnosed with coexistence of a gastric GIST, intro-abdominal and retroperitoneal tumors preoperatively.

A surgical operation was performed. Intraoperatively, a huge oval lobulated oozing soft mass (about $18.0 * 25.0 * 15.0 \mathrm{~cm}$ ) originating from the descending 

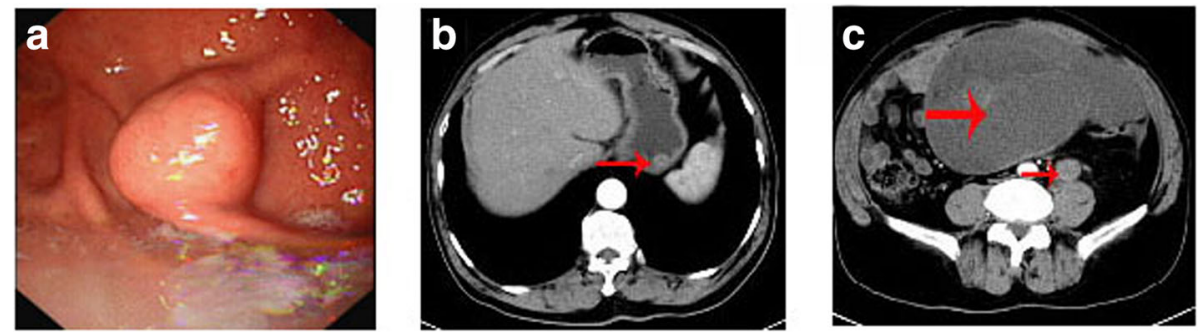

Fig. 1 a Upper gastrointestinal (GI) endoscopy showed a tumor arising from the greater curvature of gastric body and extending into the lumen. b CT scan showed a marked enhancement of polypoid mass protruding into the gastric lumen. c CT scan obtained at a lower level displayed a huge, well-circumscribed, lobulated, intro-abdominal mass and a heterogeneous, round-like mass close to the left psoas

colon mesentery was identified. A tumor (about $2.5 * 2.0 * 2.0 \mathrm{~cm})$ at the greater curvature of gastric body and a tumor (about 5.0*4.0*2.5 $\mathrm{cm}$ ) close to the left psoas were detected. Adjacent to the anterior wall of the abdominal aorta, the intro-abdominal tumor was surrounded by the small bowel and left hemicolon. The round-like lobulated retroperitoneal tumor was found among the left psoas, left iliac vessels, sigmoid colon and intro-abdominal tumor. The tumors were well-circumscribed from the surrounding organs and without signs of infiltrating tumor growth. Furthermore, the tumors were not related to the adjacent major vessels, without the detection of distant metastasis or nodal involvement. Wedge resection for gastric tumor, complete resection for intro-abdominal and retroperitoneal tumors were performed.

In histopathological examination, the intro-abdominal tumor was a myxoid liposarcoma and the retroperitoneal mass shared the same pathological type with the tumor (Fig. 2).

Further histopathological examination of the gastric carcinoma indicated a GIST of the low-risk category (mitotic index $<5$ mitoses/50 high-power fields) (Fig. 2). Immunohistochemistry displayed strong staining for c-Kit/CD117, Dog-1 and CD34, while expression of SMA and Desmin was negative (Fig. 3). Furthermore, mutations in KIT exons and PDGFRA exons were evaluated in the sample, but, nothing special was found.

Nested reverse transcription-polymeras-ase chain reaction (RT-PCR) technique was employed to detect the
FUS-CHOP mRNA expression in the formalin-fixed paraffin-embedded lipsarcoma samples. Type II FUSCHOP mRNA was successfully detected in the retroperitoneal liposarcoma (Fig. 4). The postoperative course was uneventful. Adjuvant radiotherapy was targeted to the former liposarcoma bed: Dosage in total, $50 \mathrm{~Gy}$; single dose, 1.6 Gy. The patient received CT scans twice a year, displaying no evidence of tumor recurrence in a follow up period of 15 months.

\section{Discussion}

GISTs, comprising about $0.1-3 \%$ of all gastrointestinal malignant tumors, are rare.These tumors, strongly expressing the c-KIT protein (CD117; a type III tyrosine kinase receptor encoded by the c-kit proto-ongogene), are identified as arise from interstitial cells of Cajal or their precursors [2]. As one of the most common mesenchymal neoplasms of the gastrointestinal tract, GISTs arise mainly from the stomach, small bowel and duodenum. Although GISTs usual present with alimentary tract hemorrhage, abdomen mass, abdominal pain and gastrointestinal obstruction, one-third of all cases are found incidentally. As shown in our case, based on the tumor detected during gastrointestinal endoscopy and CT scans due to the symptoms related to the huge liposarcoma, the gastric GIST could be classified as an incidental tumor.

According to the World Health Organization (WHO), lipomatous tumors were classified into well-differentiated, myxoid, pleomorphic and dedifferentiated subtypes [4]. Myxoid liposarcoma, a common subtype, is usually
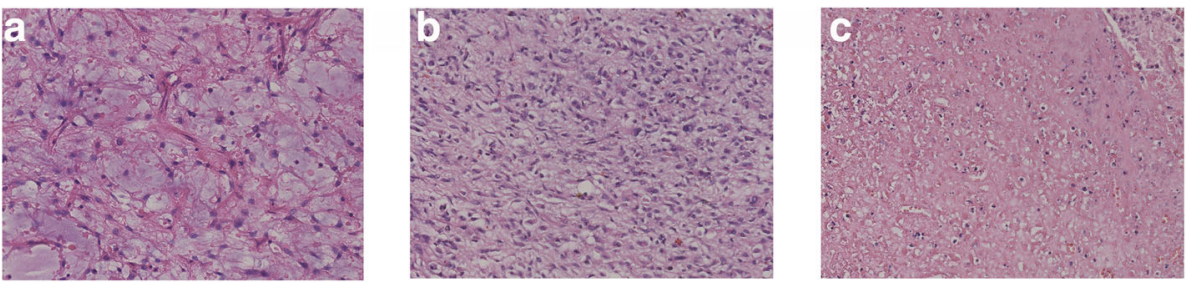

Fig. 2 a Microscopic image of GIST (× 20 magnification). b Microscopic image of intro-abdominal liposarcoma (×20 magnification). c Microscopic image of retroperitoneal liposarcoma ( $\times 20$ magnification) 

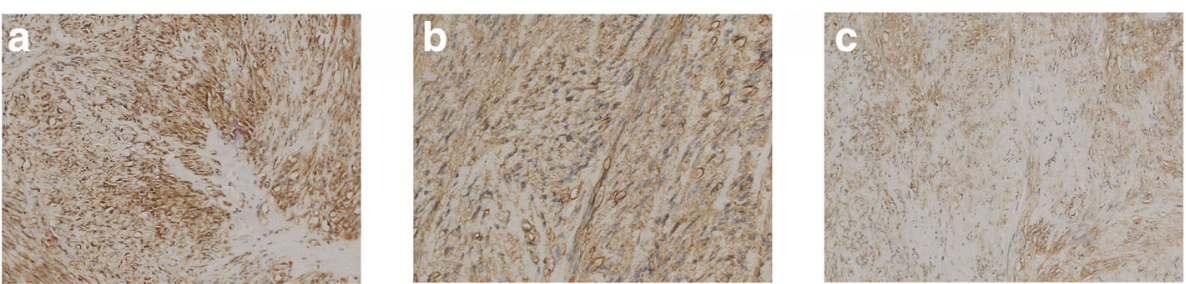

Fig. 3 The immunohistochemistry indicated strong staining for c-Kit/CD117 (a, $\times 20$ magnification), CD34 (b, $\times 20$ magnification), Dog-1 $(\mathbf{c}, \times 20$ magnification)

asymptomatic, incidentally identified, which occurs most commonly in deep soft tissues of the extremities while uncommon sites include the head and neck, thorax, and subcutis [5].The retroperitoneum is the most frequent site of liposarcoma. Up to $40 \%$ of liposarcomas occur in the intro-abdominal localization while those originated from the mesentery are rare. It is worth noting that primary retroperitoneal myxoid liposarcomas have been considered rare or even nonexistent [6], according to the recent studies. Furthermore, clinicopathologic studies confirmed that myxoid liposarcomas prefer to metastasize to deep soft tissues or bones, and the retroperitoneum stands for one of the most common metastatic locations [7]. As shown in our case, retroperitoneal liposarcoma could be considered as a metastatic mass from intro-abdominal myxoid liposarcoma.

Both GISTs and liposarcomas arise from mesenchymal tissues, coincidence of which is rarely reported. We only found two previously reported cases in the literature $[8,9]$. One case showed a gastric GIST coexistence of a retroperitoneal liposarcoma [8], while the other reported a case of coincident abdominal carcinoid tumor, GIST and well-differentiated liposarcoma in a NF1 patient [9]. Various hypotheses including gene mutations, expression of metallothioneins (MT) and influenced neighboring tissues by the same carcinogen have been used to explain the simultaneous development of a GIST and other carcinomas $[10,11]$. It is worth noting that genetic changes and polymorphisms play an important role in the development of sarcomas [12]. Furthermore, there is emerging data on genetic polymorphisms associated with susceptibility to a wide range of neoplasms $[13,14]$, which can be reasonably explained by genetic changes and polymorphisms.

Radical surgery is the main treatment for all mesenchymal tumors. Standing for the only curable chance for primary GIST, surgical resection can be achieved by only a

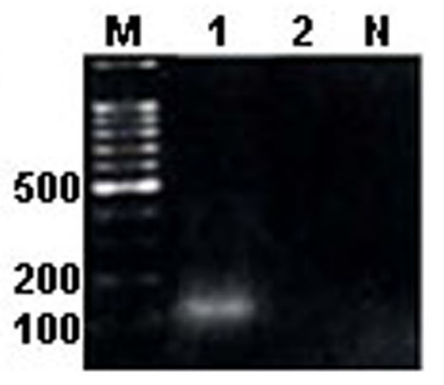

b

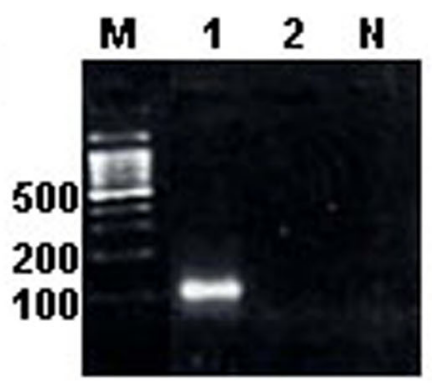

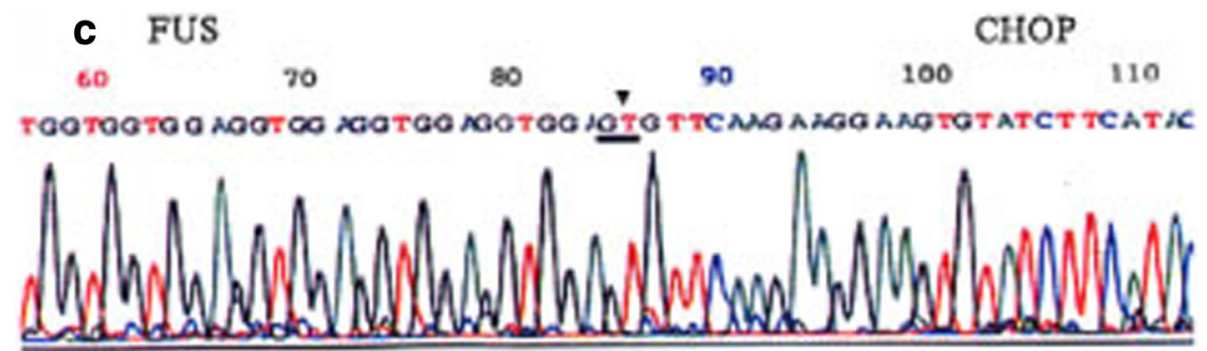

Fig. 4 M: DNA marker (DL2000), Lane 1: retroperitoneal liposarcoma, Lane 2: intro-abdominal liposarcoma, N: Negative control, $\mathbf{\nabla}$ : show the location of FUS-CHOP fusion. a Result of RT-PCR Assay of samples for $\beta$-actin mRNA. b Result of RT-PCR Assay of samples for FUS-CHOP mRNA. c Result of colonies sequencing for PCR products of FUS-CHOP mRNA 
wedge resection of the stomach or a segmental resection of the small bowel, while extensive surgery is occasionally performed for larger or poorly positioned GISTs [15]. Surgical resection is the only effective treatment for liposarcoma. During the procedure, the tissues or the organs adherent to the tumor usually need to be removed [16]. Even debulking surgery is sometimes helpful to treat with nettlesome symptoms of recurrence or un-resectable liposarcoma [17]. However, debulking surgery is not recommended in the recurrent or un-resectable GIST and for these patients, tyrosine-kinase inhibitor is a reasonable choice. In our case, the patient was diagnosed as simultaneous development of low grade gastric GIST, intro-abdominal andretroperitoneal myxoid liposarcomas. The evidence supporting the application of chemotherapy and radiotherapy for liposarcoma is limited [18]. A retrospective study showed a good local control rate for myxoid liposarcoma patients accepting surgery and radiotherapy [19], thereby, the patient was suggested to accept radiotherapy. The patient received CT scans twice a year, displaying no evidence of tumor recurrence in a follow up period of 15 months, making a long follow-up period necessary.

\section{Abbreviations}

CEA: Carcinoembryonic antigen; CT: Computed tomography; Gl: Gastrointestinal endoscopy; GIST: Gastrointestinal stromal tumor; MT: Metallothioneins; WHO: World Health Organization

\section{Acknowledgements}

We wish to thank pathologists Dr. Hai-ou Zhao for his contribution in the preparation of pathology images used in this report.

\section{Availability of data and materials}

The datasets used and/or analysed during the current study are available from the corresponding author on reasonable request.

\section{Authors' contributions}

$Y Z$ and $X W$ compiled all information relating to the patient and wrote the manuscript. QS, CX and JJ were involved in data interpretation and manuscript preparation. All authors read and approved the final manuscript.

\section{Ethics approval and consent to participate}

The study was reviewed and approved by the Yancheng City No. 1 People's Hospital Institutional Review Board.

\section{Consent for publication}

Written informed consent was obtained from the patient for publication of this case report and accompanying images.

\section{Competing interests}

No potential conflicts of interest were disclosed.

\section{Publisher's Note}

Springer Nature remains neutral with regard to jurisdictional claims in published maps and institutional affiliations.

\section{Author details}

'Department of General surgery, Yancheng City No.1 people's hospital, Yancheng, Jiangsu Province 224005, China. ${ }^{2}$ Department of Gastroenterology, Yancheng City No.1 people's hospital, 16 Yuehe Road, Yancheng, Jiangsu Province 224005, China. ${ }^{3}$ Department of Radiology,
Yancheng City No.1 people's hospital, Yancheng, Jiangsu Province 224005, China. ${ }^{4}$ Department of Pathology, Yancheng City No.1 people's hospital, Yancheng, Jiangsu Province 224005, China. ${ }^{5}$ Department of Nephrology, Yancheng City No.1 people's hospital, Yancheng, Jiangsu Province 224005, China.

Received: 17 November 2017 Accepted: 1 October 2018

Published online: 11 October 2018

\section{References}

1. Theodosopoulos T, Dellaportas D, Psychogiou V, Gennatas K, Kondi-Pafiti A Gkiokas G, Papaconstantinou I, Polymeneas G. Synchronous gastric adenocarcinoma and gastrointestinal stromal tumor (GIST) of the stomach: a case report. World J Surg Oncol. 2011;9:60.

2. Zhou Y, Wu XD, Shi Q, Jia J. Coexistence of gastrointestinal stromal tumor esophageal and gastric cardia carcinomas. World J Gastroenterol. 2013; 19(12):2005-8.

3. Cerullo G, Marrelli D, Rampone B, Perrotta E, Caruso S, Roviello F. Giant liposarcoma of the mesentery. Report of a case. Ann Ital Chir. 2007:78(5):443-5.

4. Meher S, Mishra TS, Rath S, Sasmal PK, Mishra P, Patra S. Giant dedifferentiated liposarcoma of small bowel mesentery: a case report. World J Surg Oncol. 2016:14(1):250.

5. Setsu N, Miyake M, Wakai S, Nakatani F, Kobayashi E, Chuman H, Hiraoka N, Kawai A, Yoshida A. Primary retroperitoneal myxoid liposarcomas. Am J Surg Pathol. 2016;40(9):1286-90

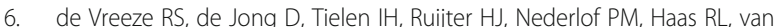
Coevorden F. Primary retroperitoneal myxoid/round cell liposarcoma is a nonexisting disease: an immunohistochemical and molecular biological analysis. Mod Pathol. 2009;22:223-31.

7. Pearlstone DB, Pisters PW, Bold RJ, Feig BW, Hunt KK, Yasko AW, Patel S, Pollack A, Benjamin RS, Pollock RE. Patterns of recurrence in extremity liposarcoma: implications for staging and follow-up. Cancer. 1999;85:85-92.

8. Arend J, Kuester D, Roessner A, Lippert H, Meyer F. Gastric high-risk gist and retroperitoneal liposarcoma - a challenging combination of two mesenchymal tumor lesions with regard to diagnosis and treatment. Pol Przegl Chir. 2013; 85(5):284-8.

9. James AW, Chang L, Genshaft S, Dry SM. Coincident liposarcoma, carcinoid and gastrointestinal stromal tumor complicating type 1 neurofibromatosis: Case report and literature review. J Orthop. 2013;12(Suppl 1):S111-6.

10. Rauf F, Ahmad Z, Muzzafar S, Hussaini AS. Synchronous occurrence of gastrointestinal stromal tumor and gastric adenocarcinoma: a case report. J Pak Med Assoc. 2006:56:184-6.

11. Nemes C, Rogojan L, Surdea-Blaga T, Seicean A, Dumitrascu DL, Ciuce C. Gastrointestinal stromal tumor (GIST) associated with synchronous Colon adenocarcinoma- a case report. J Gastrointestin Liver Dis. 2012;21(1):101-3.

12. Soini $Y$. Epigenetic and genetic changes in soft tissue sarcomas: a review. APMIS. 2016;124(11):925-34.

13. Zhou S, Ruan Y, Yu H, Chen Y, Yao Y, Ma Y, Gao Y. Functional IL-23R rs10889677 genetic polymorphism and risk of multiple solid tumors: a meta-analysis. PLoS One. 2013:8(11):e80627.

14. Jia Y, Xie X, Shi X, Li S. Associations of common IL-4 gene polymorphisms with cancer risk: A meta-analysis. Mol Med Rep. 2017;16(2):1927-45.

15. Chaudhry UI, DeMatteo RP. Advances in the surgical Management of Gastrointestinal Stromal Tumor (GIST). Adv Surg. 2011:45:197-209.

16. Winn B, Gao J, Akbari H, Bhattacharya B. Dedifferentiated liposarcoma arising from the sigmoid mesocolon: a case report. World J Gastroenterol. 2007:13(30):4147-8.

17. Takeda K, Aimoto T, Yoshioka M, Nakamura Y, Yamahatsu K, Ishiwata T, Naito Z Miyashita M, Uchida E. Dedifferentiated liposarcoma arising from the mesocolon ascendens: report of a case. J Nippon Med Sch. 2012;79(5):385-90.

18. Perez EA, Gutierrez JC, Moffat FL Jr, Franceschi D, Livingstone AS, Spector SA, Levi JU, Sleeman D, Koniaris LG. Retroperitoneal and truncal sarcomas: prognosis depends upon type not location. Ann Surg Oncol. 2007;14:1114-22.

19. Guadagnolo BA, Zagars GK, Ballo MT, Patel SR, Lewis VO, Benjamin RS, Pollock RE. Excellent local control rates and distinctive patterns of failure in myxoid liposarcoma treated with conservation surgery and radiotherapy. Int J Radiat Oncol Biol Phys. 2008;70:760-5. 